\title{
Disk-like Chemistry of the Triangulum-Andromeda Overdensity as Seen by APOGEE
}

\author{
Christian R. Hayes $^{1}$ (1), Steven R. Majewski ${ }^{1}$ (1), Sten Hasselquist ${ }^{2}$, Rachael L. Beaton ${ }^{3,4,21,22}{ }^{\oplus}$, Katia Cunha ${ }^{5,6}$, Verne V. Smith ${ }^{7}$, \\ Adrian M. Price-Whelan ${ }^{3}$ (1) Borja Anguiano ${ }^{1,8}$, Timothy C. Beers ${ }^{9}$ (i) , Ricardo Carrera ${ }^{10}$ (1) J. G. Fernández-Trincado ${ }^{11,12}$, \\ Peter M. Frinchaboy ${ }^{13}{ }^{1}$, D. A. García-Hernández ${ }^{14,15}$, Richard R. Lane ${ }^{16,17}$, David L. Nidever ${ }^{7,18}$ (1) Christian Nitschelm ${ }^{19}$, \\ Alexandre Roman-Lopes ${ }^{20}$ (D), and Olga Zamora ${ }^{14,15}$ \\ ${ }^{1}$ Department of Astronomy, University of Virginia, Charlottesville, VA 22904-4325, USA; crh7gs@ virginia.edu \\ ${ }^{2}$ Department of Astronomy, New Mexico State University, Las Cruces, NM 88003, USA \\ ${ }^{3}$ Department of Astrophysical Sciences, Princeton University, 4 Ivy Lane, Princeton, NJ 08544, USA \\ ${ }^{4}$ The Observatories of the Carnegie Institution for Science, 813 Santa Barbara Street, Pasadena, CA 91101, USA \\ ${ }^{5}$ Observatório Nacional, 77 Rua General José Cristino, Rio de Janeiro, 20921-400, Brazil \\ ${ }^{6}$ Steward Observatory, University of Arizona, 933 North Cherry Avenue, Tucson, AZ 85721, USA \\ ${ }^{7}$ National Optical Astronomy Observatory, 950 North Cherry Avenue, Tucson, AZ 85719, USA \\ ${ }^{8}$ Department of Physics and Astronomy, Macquarie University, North Ryde, NSW 2109, Australia \\ ${ }^{9}$ Department of Physics, University of Notre Dame, and JINA Center for the Evolution of the Elements, Notre Dame, IN 46556 USA \\ ${ }^{10}$ Astronomical Observatory of Padova, National Institute of Astrophysics, Vicolo Osservatorio 5-35122-Padova, Italy \\ ${ }^{11}$ Departamento de Astronomía, Universidad de Concepción, Casilla 160-C, Concepción, Chile \\ ${ }^{12}$ Institut Utinam, CNRS UMR6213, Univ. Bourgogne Franche-Comté, OSU THETA, Observatoire de Besançon, BP 1615, F-25010 Besançon Cedex, France \\ ${ }^{13}$ Department of Physics and Astronomy, Texas Christian University, Fort Worth, TX 76129, USA \\ ${ }^{14}$ Instituto de Astrofísica de Canarias (IAC), Vía Láctea, E-38205 La Laguna, Tenerife, Spain \\ ${ }_{16}^{15}$ Departamento de Astrofísica, Universidad de La Laguna (ULL), E-38206 La Laguna, Tenerife, Spain \\ ${ }^{16}$ Millennium Institute of Astrophysics, Av. Vicuña Mackenna 4860, 782-0436 Macul, Santiago, Chile \\ ${ }^{17}$ Instituto de Astrofísica, Pontificia Universidad Católica de Chile, Av. Vicuña Mackenna 4860, 782-0436 Macul, Santiago, Chile \\ ${ }_{18}$ Department of Physics, Montana State University, P.O. Box 173840, Bozeman, MT 59717-3840, USA \\ ${ }^{19}$ Unidad de Astronomía, Universidad de Antofagasta, Avenida Angamos 601, Antofagasta 1270300, Chile \\ ${ }^{20}$ Departamento de Física, Facultad de Ciencias, Universidad de La Serena, Cisternas 1200, La Serena, Chile \\ Received 2018 April 18; revised 2018 May 8; accepted 2018 May 8; published 2018 May 21
}

\begin{abstract}
The nature of the Triangulum-Andromeda (TriAnd) system has been debated since the discovery of this distant, low-latitude Milky Way (MW) overdensity more than a decade ago. Explanations for its origin are either as a halo substructure from the disruption of a dwarf galaxy, or a distant extension of the Galactic disk. We test these hypotheses using the chemical abundances of a dozen TriAnd members from the Sloan Digital Sky Survey-IV's (SDSS-IV's) 14th Data Release (DR14) of Apache Point Observatory Galactic Evolution Experiment (APOGEE) data to compare to APOGEE abundances of stars with similar metallicity from both the Sagittarius (Sgr) dSph and the outer MW disk. We find that TriAnd stars are chemically distinct from Sgr across a variety of elements, $(\mathrm{C}+\mathrm{N})$, $\mathrm{Mg}, \mathrm{K}, \mathrm{Ca}, \mathrm{Mn}$, and $\mathrm{Ni}$, with a separation in $[\mathrm{X} / \mathrm{Fe}]$ of about 0.1 to 0.4 dex depending on the element. Instead, the TriAnd stars, with a median metallicity of about -0.8 , exhibit chemical abundance ratios similar to those of the lowest metallicity $([\mathrm{Fe} / \mathrm{H}] \sim-0.7)$ stars in the outer Galactic disk, and are consistent with expectations of extrapolated chemical gradients in the outer disk of the MW. These results suggest that TriAnd is associated with the MW disk, and, therefore, that the disk extends to this overdensity-i.e., past a Galactocentric radius of $24 \mathrm{kpc}$ -albeit vertically perturbed about $7 \mathrm{kpc}$ below the nominal disk midplane in this region of the Galaxy.
\end{abstract}

Key words: Galaxy: disk - Galaxy: evolution - Galaxy: halo - Galaxy: structure - stars: abundances

Supporting material: machine-readable table

\section{Introduction}

Several overdensities discovered toward the outer disk of the Milky Way (MW) have origins that are still not understood. These include "Triangulum-Andromeda" (TriAnd; Majewski et al. 2004; Rocha-Pinto et al. 2004), which is a low-latitude, distant ( $\sim 20 \mathrm{kpc})$, and kinematically cold $\left(\sigma_{\mathrm{LOS}} \sim 25 \mathrm{~km} \mathrm{~s}^{-1}\right)$ cloud of stars (Sheffield et al. 2014). Theories to explain TriAnd's observed properties include that it (1) could be tidal debris from a disrupted dwarf galaxy (Deason et al. 2014; Sheffield et al. 2014), or (2) represents part of an extended and perturbed MW disk, perhaps a trough in a series of midplane oscillations (Price-Whelan et al. 2015; Xu et al. 2015; Li et al. 2017). Recent simulations have illustrated that large,

\footnotetext{
${ }^{21}$ Hubble Fellow.

22 Carnegie-Princeton Fellow.
}

non-axisymmetric, vertical oscillations can be excited in the outer disk due to the interaction of the Sagittarius dwarf spheroidal galaxy (Sgr dSph) with the MW, thus reproducing structures reminiscent of TriAnd (and other overdensities; Laporte et al. 2017). These differing origin scenarios should impart different chemical signatures to TriAnd stars. For example, if TriAnd is the result of a perturbation to the outer Galactic disk, its chemical abundance patterns should resemble that of known outer disk stars, whereas tidal debris should share the chemistry seen in dwarf galaxies.

To date, chemical studies of TriAnd have reached differing conclusions about its origin. The first high-resolution spectroscopic study of TriAnd stars by Chou et al. (2011) focused on the elements $\mathrm{Ti}, \mathrm{Y}$, and $\mathrm{La}$, and found TriAnd had some chemical differences from MW disk stars in the solar neighborhood, and suggested a dwarf galaxy origin. On the other hand, the recent study of $\mathrm{O}, \mathrm{Na}, \mathrm{Mg}, \mathrm{Ti}, \mathrm{Ba}$, and $\mathrm{Eu}$ 
Table 1

Properties of TriAnd Stars

\begin{tabular}{|c|c|c|c|c|c|}
\hline Column & Column Label & Column Description & Column & Column Label & Column Description \\
\hline 1 & APOGEE & APOGEE Star ID & 17 & Vturb & Microturbulent velocity $\left(\mathrm{km} \mathrm{s}^{-1}\right)$ \\
\hline 2 & RAdeg & Right Ascension (decimal degrees) & 18 & Vmacro & Macroturbulent velocity $\left(\mathrm{km} \mathrm{s}^{-1}\right)$ \\
\hline 3 & DEdeg & Declination (decimal degrees) & 19 & {$[\mathrm{Fe} / \mathrm{H}]$} & Log abundance, $[\mathrm{Fe} / \mathrm{H}]$ \\
\hline 4 & GLON & Galactic Longitude (decimal degrees) & 20 & $\mathrm{e} \_[\mathrm{Fe} / \mathrm{H}]$ & Uncertainty in $[\mathrm{Fe} / \mathrm{H}]$ \\
\hline 5 & GLAT & Galactic Latitude (decimal degrees) & 21 & {$[\mathrm{CN} / \mathrm{Fe}]$} & Log abundance, $[(\mathrm{C}+\mathrm{N}) / \mathrm{Fe}]$ \\
\hline 6 & Jmag & 2MASS J magnitude & 22 & e_[CN/Fe] & Uncertainty in $[(\mathrm{C}+\mathrm{N}) / \mathrm{Fe}]$ \\
\hline 7 & Hmag & 2MASS H magnitude & 23 & {$[\mathrm{Mg} / \mathrm{Fe}]$} & Log abundance, $[\mathrm{Mg} / \mathrm{Fe}]$ \\
\hline 8 & Kmag & 2MASS Ks magnitude & 24 & e_ $[\mathrm{Mg} / \mathrm{Fe}]$ & Uncertainty on $[\mathrm{Mg} / \mathrm{Fe}]$ \\
\hline 9 & Dist & Heliocentric distance $(\mathrm{kpc})$ & 25 & {$[\mathrm{~K} / \mathrm{Fe}]$} & Log abundance, $[\mathrm{K} / \mathrm{Fe}]$ \\
\hline 10 & e_Dist & Uncertainty in distance $(\mathrm{kpc})$ & 26 & $\mathrm{e} \_[\mathrm{K} / \mathrm{Fe}]$ & Uncertainty on $[\mathrm{K} / \mathrm{Fe}]$ \\
\hline 11 & HRV & Heliocentric radial velocity $\left(\mathrm{km} \mathrm{s}^{-1}\right)$ & 27 & {$[\mathrm{Ca} / \mathrm{Fe}]$} & Log abundance, $[\mathrm{Ca} / \mathrm{Fe}]$ \\
\hline 12 & e_HRV & Radial velocity uncertainty $\left(\mathrm{km} \mathrm{s}^{-1}\right)$ & 28 & $\mathrm{e} \_[\mathrm{Ca} / \mathrm{Fe}]$ & Uncertainty on $[\mathrm{Ca} / \mathrm{Fe}]$ \\
\hline 13 & Teff & Effective surface temperature (K) & 29 & {$[\mathrm{Mn} / \mathrm{Fe}]$} & Log abundance, $[\mathrm{Mn} / \mathrm{Fe}]$ \\
\hline 14 & e_Teff & Uncertainty in $T_{\text {eff }}(\mathrm{K})$ & 30 & $\mathrm{e} \_[\mathrm{Mn} / \mathrm{Fe}]$ & Uncertainty on $[\mathrm{Mn} / \mathrm{Fe}]$ \\
\hline 15 & $\log g$ & Surface gravity & 31 & {$[\mathrm{Ni} / \mathrm{Fe}]$} & Log abundance, $[\mathrm{Ni} / \mathrm{Fe}]$ \\
\hline 16 & e_logg & Uncertainty in $\log g$ & 32 & e_[Ni/Fe] & Uncertainty on $[\mathrm{Ni} / \mathrm{Fe}]$ \\
\hline
\end{tabular}

Note. Null entries are given values of -9999 .

(This table is available in its entirety in machine-readable form.)

abundance ratios in TriAnd stars indicate that it is chemically consistent with the MW disk rather than a dwarf galaxy (Bergemann et al. 2018). Further chemical study of TriAnd to resolve such discrepancies is clearly warranted.

The Apache Point Observatory Galactic Evolution Experiment (APOGEE; Majewski et al. 2017) provides such an opportunity. APOGEE is a high-resolution $(R \sim 22,500)$ spectroscopic survey of Galactic stellar populations with $H$-band sensitivity that is well suited to the exploration of highly extinguished low-latitude targets, such as the TriAnd overdensity and the outer disk. Selecting from the $\sim 263,000$ stars observed with the SDSS $2.5 \mathrm{~m}$ telescope (Gunn et al. 2006) and analyzed by APOGEE in the 14th Data Release (DR14; Abolfathi et al. 2018) of the Sloan Digital Sky Survey-IV (SDSS-IV; Blanton et al. 2017), we use the abundances of six APOGEE-measured elements to compare TriAnd red giants to outer disk and Sgr dSph stars, and demonstrate that the TriAnd chemistry is more consistent with an extrapolation of outer MW disk chemical gradients than the abundance patterns of a prototypical dwarf galaxy of similar enrichment.

\section{Data}

Details of the APOGEE survey and data reduction pipeline can be found in Majewski et al. (2017) and Nidever et al. (2015), respectively. Here we use the SDSS-IV DR14 calibrated stellar parameters and chemical abundances derived from the APOGEE Stellar Parameter and Chemical Abundance Pipeline (ASPCAP; García Pérez et al. 2016). To insure that we are considering the most reliably determined stellar parameters, we remove stars flagged $^{23}$ with the STARFLAGS BAD_PIXELS, VERY_BRIGHT_ NEIGHBOR, or LOW_SNR set, or any stars with the ASPCAPFLAGS, ROTATION_WARN or STAR_BAD. We also restrict analysis to stars with small visit-to-visit velocity scatter, $V_{\text {scatter }} \leqslant 1 \mathrm{~km} \mathrm{~s}^{-1}$, low velocity uncertainty, $V_{\text {err }} \leqslant 0.2 \mathrm{~km} \mathrm{~s}^{-1}$, and signal-to-noise ratio $(\mathrm{S} / \mathrm{N})>80$ to remove stars with

\footnotetext{
${ }^{23}$ A description of these flags can be found in the online SDSS DR14 bitmask documentation (http://www.sdss.org/dr14/algorithms/bitmasks/).
}

ASPCAP-analyzed spectra that may be of lower quality. Finally, we focus on stars in effective temperature ranges, between $3700 \mathrm{~K}$ and $5500 \mathrm{~K}$, where stellar parameters and chemical abundances are reliably and consistently determined.

In this high-quality sample, we have $12 \mathrm{M}$ giants that were identified by Sheffield et al. (2014) as TriAnd members from their photometry and cold kinematics $\left(\sigma \sim 25 \mathrm{~km} \mathrm{~s}^{-1}\right)$ and were deliberately targeted in APOGEE-2 (Zasowski et al. 2017). The APOGEE-measured properties of these TriAnd stars are given in Table 1. Several studies have suggested that TriAnd may separate into two features, TriAnd 1 and TriAnd2, that coincide on the sky but lie at photometrically determined heliocentric distances $\sim 20 \mathrm{kpc}$ and $\sim 28 \mathrm{kpc}$, respectively (Martin et al. 2007). However, because these features were shown to overlap considerably in spectrophotometric distance, radial velocity, and metallicity (Sheffield et al. 2014), and we only have two stars classified as TriAnd2 members, we treat them here as a single overdensity. For a comparison, we use $\mathrm{Sgr} \mathrm{dSph}$ because this dwarf galaxy is sufficiently enriched to have a considerable M giant population, like TriAnd. To do so, we use a set of 69 Sgr dSph members confirmed by Hasselquist et al. (2017) and satisfy our quality criteria.

We also compile a representative comparison sample of outer disk stars from APOGEE. To do so, we use spectrophotometric distances calculated by Queiroz et al. (2018) using DR14 ASPCAP stellar parameters and their Bayesian StarHorse code. Because not all of these distances are reliable, we only use stars that are not flagged with HIGH_EXTINCTION_WARN, NUMMODELS_BAD, or EXTINCTION_BAD_BRIGHT2MASS, which may have erroneous distance estimates due to poor extinction corrections or lack available stellar models to determine a reliable distance. Because we will determine metallicity and chemical gradients in the outer disk to compare to the chemistry of TriAnd stars, we also want stars with relatively accurate StarHorse distances, and remove stars with $\sigma_{d}>0.5 \mathrm{kpc}$ on their posterior distance distribution. These distances are converted to Galactocentric coordinates assuming $R_{\mathrm{GC}, \odot}=8 \mathrm{kpc}$, and the Galactic distribution of these APOGEE stars is shown in Figure 1, along with the TriAnd stars with StarHorse distances. Finally, we 

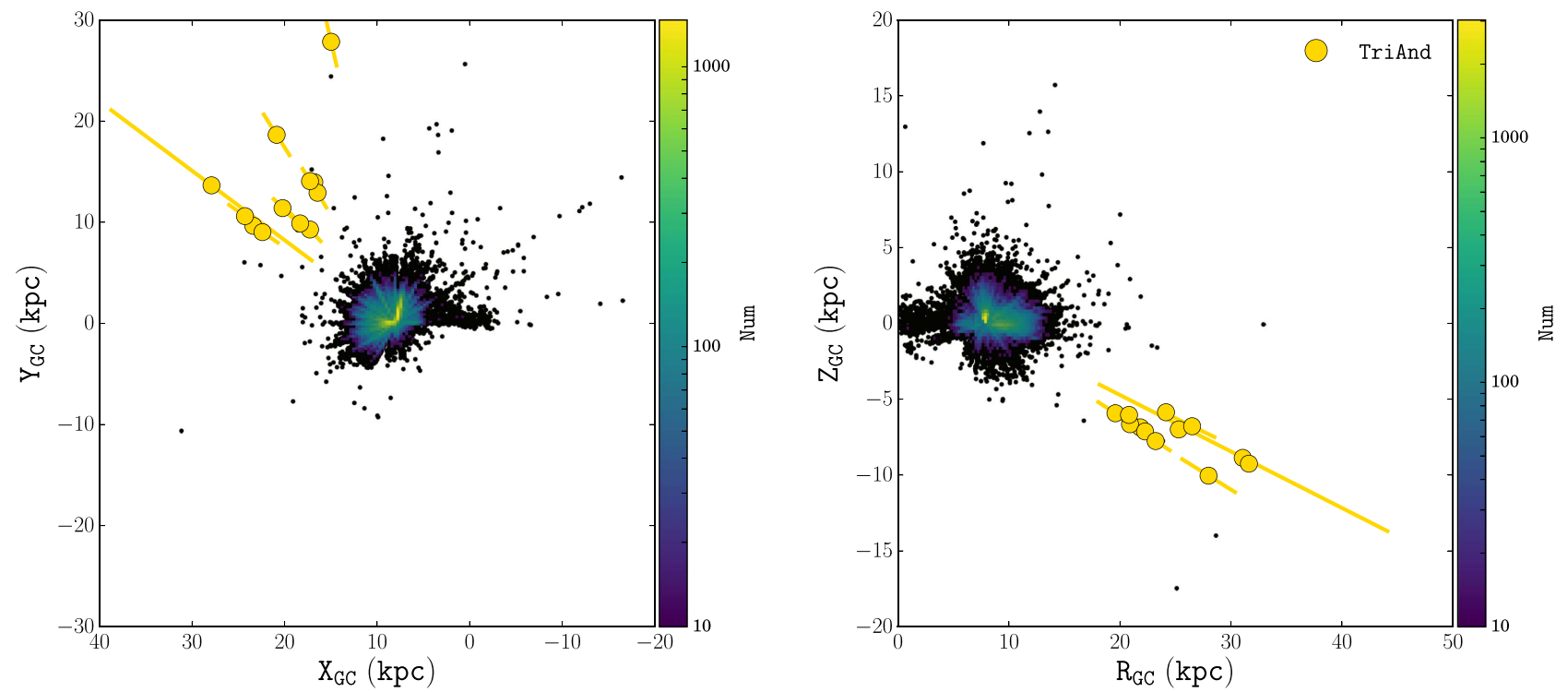

Figure 1. Spatial distribution of the high-quality APOGEE stars (before outer disk selection, black points) and TriAnd stars (gold circles), showing the reported $1 \sigma$ distance uncertainty for the TriAnd stars. Left: stellar distribution projected onto the Galactic plane. Right: distribution azimuthally collapsed onto the cylindrical $R_{\mathrm{GC}}-Z_{\mathrm{GC}}$ plane.

form our "outer disk sample" from this high-quality set of MW stars by selecting those with cylindrical Galactocentric radii $R_{\mathrm{GC}}>9 \mathrm{kpc}$ and midplane distances $|Z|<1.0 \mathrm{kpc}$.

\section{Results and Analysis}

Using the reliable spectrophotometric distances from StarHorse, our TriAnd sample (with median distance uncertainties of $2 \mathrm{kpc}$ ) is centered at a median distance of $\sim 18 \mathrm{kpc}$ with a $1 \sigma$ spread of $4 \mathrm{kpc}$. This is consistent with past distances found for TriAnd, e.g., the $18.2 \mathrm{kpc}$ distance (Sheffield et al. 2014) used to select the "TriAnd1" members that dominate our sample here. This puts the TriAnd sample at a median Galactocentric radius of $\sim 24 \mathrm{kpc}\left(1-\sigma_{\mathrm{R}}\right.$ spread of $4 \mathrm{kpc})$ and below the disk by $\sim 7 \mathrm{kpc}\left(1-\sigma_{\mathrm{Z}}\right.$ spread of $\left.1 \mathrm{kpc}\right)$.

While a few of the TriAnd stars do not have reliable spectrophotometric distances from StarHorse, they were selected by Sheffield et al. (2014) in color-magnitude to fall along the red giant branches of $8 \mathrm{Gyr} / 10 \mathrm{Gyr}-0.8 /-1.0$ metallicity isochrones at heliocentric distances around $18.2 \mathrm{kpc} / 27.5 \mathrm{kpc}$ for TriAnd1/TriAnd2. Figure 2 shows that TriAnd stars have effective temperatures and surface gravities of cool red giants, supporting the isochrone-based distances used by Sheffield et al. (2014).

As shown below, we can perform a more robust analysis by comparing to a large sample of outer disk stars spanning a considerable range of Galactocentric radii. To achieve this, we have not restricted the outer disk sample to cover the $T_{\text {eff }}$ and $\log g$ range of the TriAnd and Sgr dSph samples. However, to ensure that this does not affect our chemical abundance comparison, we examined the abundance distributions of relevant chemical elements for outer disk stars that are warmer and cooler than $4250 \mathrm{~K}$ to verify that there were no significant differences in their abundance patterns at a level that affects our conclusions about TriAnd. Because our TriAnd and Sgr dSph samples cover nearly the same stellar parameter space, their comparison should be even more robustly reliable.

Despite its large size (21,868 stars), our APOGEE-based disk sample (targeted to minimize selection biases; Zasowski

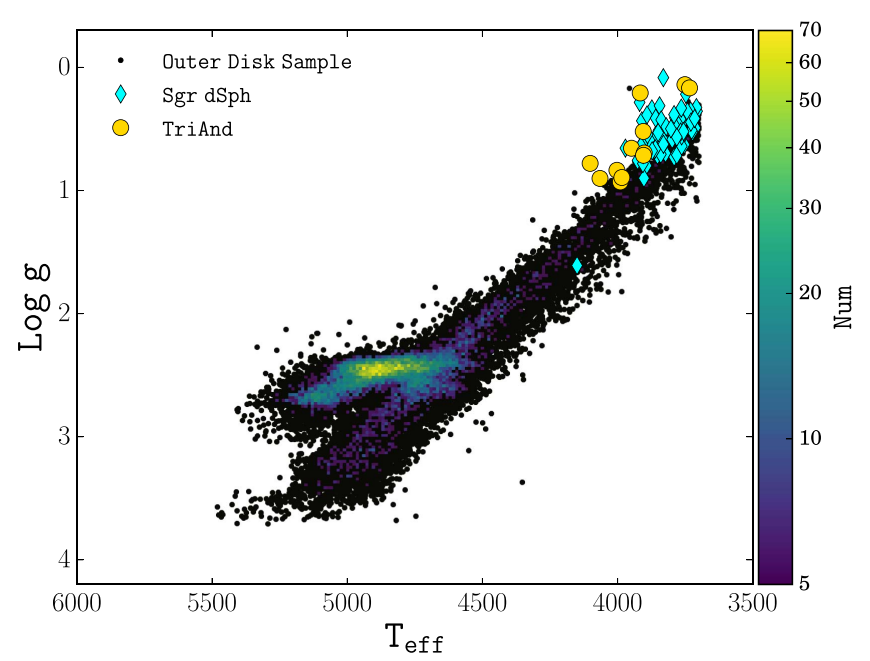

Figure 2. Spectroscopic Hertzsprung-Russell diagram of APOGEE-derived $\log g$ vs. $T_{\text {eff }}$ for the outer disk (black points with a $2 \mathrm{D}$ histogram where densely populated), TriAnd (gold circles), and Sgr dSph (cyan diamonds) samples.

et al. 2013, 2017) does not extend to the distance of the TriAnd stars and has few stars beyond $R_{\mathrm{GC}}>15 \mathrm{kpc}$. However, if the abundances of Galactic disk stars follow relatively wellbehaved radial metallicity and chemical gradients, we can extrapolate those trends to establish the abundances expected for the disk at the distance of TriAnd. To illustrate this, Figure 3 shows the $[\mathrm{Mg} / \mathrm{Fe}]-[\mathrm{Fe} / \mathrm{H}]$ plane for outer disk stars with $[\mathrm{Mg} / \mathrm{Fe}]$ and $[\mathrm{Fe} / \mathrm{H}]$ uncertainties less than $0.1 \mathrm{dex}$, subdivided into samples lying within $1 \mathrm{kpc}$-wide annuli spanning Galactocentric radii from $R_{\mathrm{GC}}=9 \mathrm{kpc}$ to $15 \mathrm{kpc}$. Within each annulus we calculate the median abundance of the outer disk sample, and can see that there are clear trends in both $[\mathrm{Fe} / \mathrm{H}]$ and $[\mathrm{Mg} / \mathrm{Fe}]$ with Galactocentric radius.

Figure 3 strikingly demonstrates that the $[\mathrm{Mg} / \mathrm{Fe}]-[\mathrm{Fe} / \mathrm{H}]$ distribution for TriAnd stars occupies a region of this parameter space that is consistent with a metal-poor extrapolation of the 


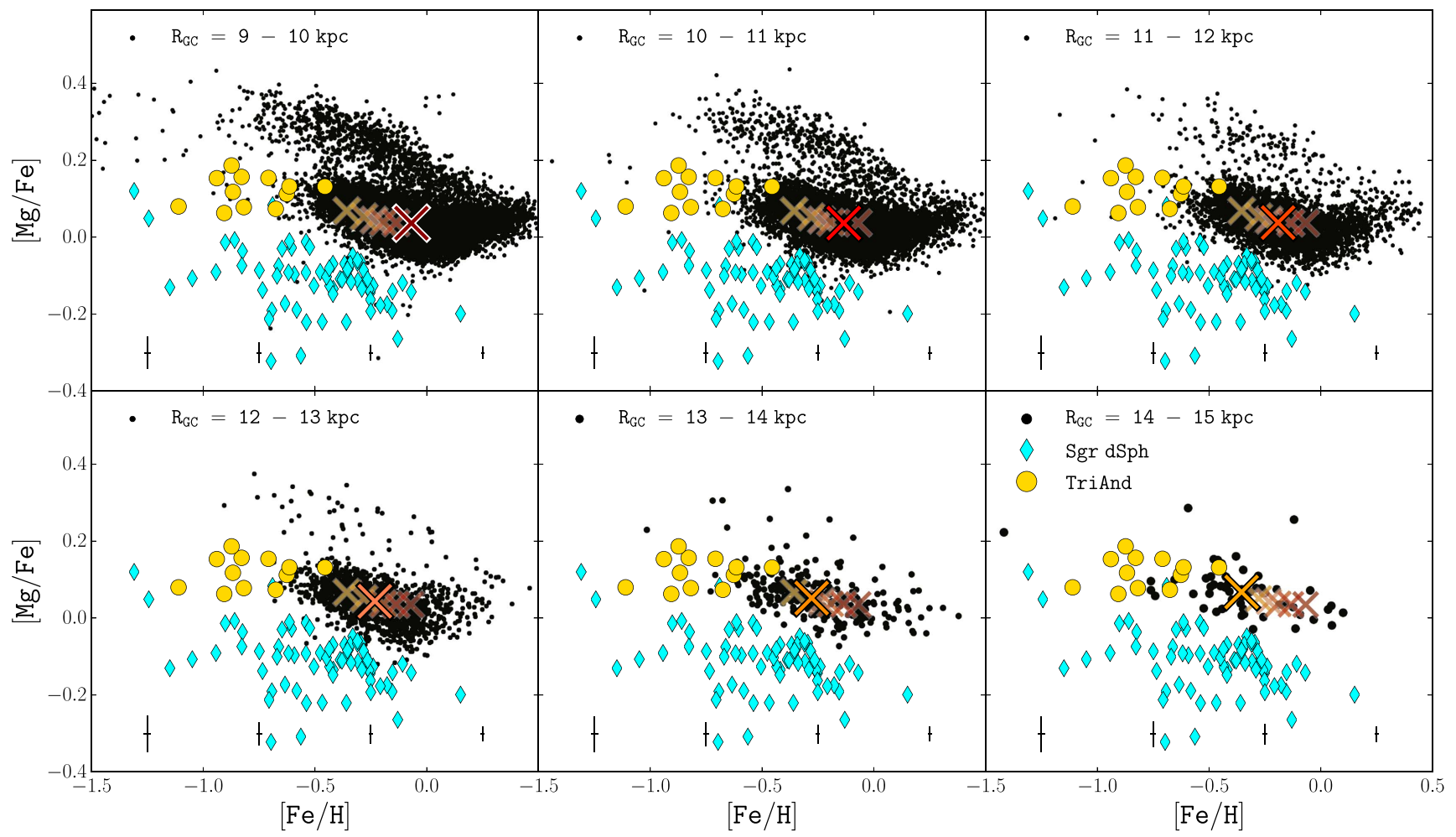

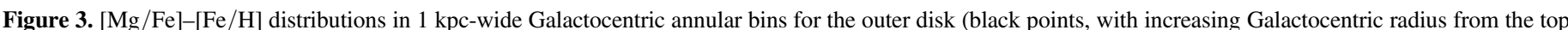

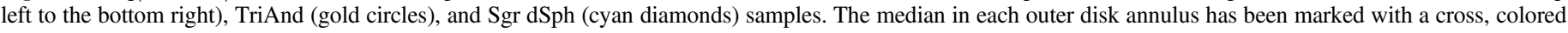

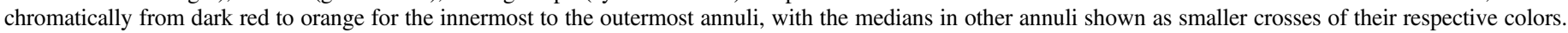
The plotted error bars show the median internal abundance uncertainties in 0.5 dex-wide metallicity bins.

outer disk trend to larger radius. Moreover, the TriAnd stars are enhanced in $[\mathrm{Mg} / \mathrm{Fe}]$ relative to $\mathrm{Sgr} \mathrm{dSph}$ stars of similar metallicity.

APOGEE enables similar comparisons of these samples in multiple chemical dimensions. Figure 4 presents the chemical abundance distributions of the TriAnd, outer disk, and Sgr dSph samples (showing only stars with both $\sigma[\mathrm{X} / \mathrm{Fe}]$ and $\sigma$ $[\mathrm{Fe} / \mathrm{H}]<0.1 \mathrm{dex})$ for a set of elements that are formed in a variety of nucleosynthetic processes: the $\alpha$-elements $\mathrm{Mg}$ and $\mathrm{Ca}$, the odd- $Z$ element $\mathrm{K}$, the iron-peak elements $\mathrm{Mn}$ and $\mathrm{Ni}$, and the sum of $\mathrm{C}$ and $\mathrm{N}$ (surface abundances of $\mathrm{C}$ and $\mathrm{N}$ are altered during dredge-up and mixing in red giants, but their sum is effectively conserved; see Martig et al. 2016, and references therein). This subset of APOGEE-measured elements were specifically chosen because they do not exhibit different abundance trends in warm and cool outer disk stars, and are measured with low uncertainties.

In these other chemical planes, as in Figure 3, the TriAnd stars tend to overlap and extend the sequence of the outer disk stars at metallicities of $[\mathrm{Fe} / \mathrm{H}] \sim-0.7$. Moreover, the radial gradient of the outer disk, as measured by the median chemistry in $1 \mathrm{kpc}$ annular rings (also as in Figure 3), approaches the chemical abundances of TriAnd stars at increasing radii. We can extrapolate these trends to the distance of TriAnd to estimate the expected abundances for outer disk stars at that location. While the shape of the $[\mathrm{X} / \mathrm{Fe}]-[\mathrm{Fe} / \mathrm{H}]$ distribution of the outer disk sample is complex within each annulus, as seen in Figure 3, the median chemistry of the distributions varies roughly linearly with Galactocentric radius. Thus, we fit the annular median abundances linearly in Galactocentric radius to find a metallicity gradient of $\partial[\mathrm{Fe} / \mathrm{H}] / \partial R_{\mathrm{GC}}=-0.051 \pm$ $0.005 \mathrm{dex} \mathrm{kpc}{ }^{-1}$ and $\partial[\mathrm{X} / \mathrm{Fe}] / \partial R_{\mathrm{GC}}$ gradients for $((\mathrm{C}+\mathrm{N}), \mathrm{Mg}$,
$\mathrm{K}, \mathrm{Ca}, \mathrm{Mn}, \mathrm{Ni})=(0.002 \pm 0.001,0.006 \pm 0.001,0.005 \pm 0.001$, $0.009 \pm 0.001,-0.001 \pm 0.001,0.003 \pm 0.002) \mathrm{dex} \mathrm{kpc}^{-1}$.

\section{Discussion}

Figure 4 demonstrates that, when extrapolated to the distance of TriAnd, the outer disk chemistry generally matches that of the TriAnd stars, which suggests that they are associated. For $[\mathrm{Ca} / \mathrm{Fe}]$ the predicted median disk chemistry lies at the edge of the TriAnd distribution, which appears to indicate that a linear extrapolation of the disk $\mathrm{Ca}$ gradient may not be appropriate. We note that the flattening of the $[\mathrm{Ca} / \mathrm{Fe}]$ trend in the outer disk at the largest radii seems astrophysically significant, and in comparison with the nearly constant gradient in the lower-mass alpha-element, $\mathrm{Mg}$, may be reflecting radial or time variations in the initial mass function or star formation history of the disk.

In contrast to the agreement with radial extrapolations of outer disk chemistry, the abundance patterns of the TriAnd stars are distinct from those of Sgr dSph stars, despite their similar metallicities. If the $\mathrm{Sgr} \mathrm{dSph}$ is representative of the chemistry of relatively enriched tidal debris falling into the MW, then the chemical differences between TriAnd and Sgr $\mathrm{dSph}$ supports the notion that TriAnd is not tidal debris, at least from this type of dwarf galaxy. Another example of a relatively enriched dwarf galaxy is the Large Magellanic Cloud (LMC). While the $\alpha$-element abundances of the LMC overlap somewhat with the metal-poor stars in the thin disk (and thus with TriAnd), the LMC exhibits low $\mathrm{Ni}$ abundances, with $[\mathrm{Ni} / \mathrm{Fe}]$ $\sim-0.2$ (van der Swaelmen et al. 2013), about 0.3 dex lower than the $[\mathrm{Ni} / \mathrm{Fe}]$ ratios found here in TriAnd stars. Thus, the LMC provides another example of a dwarf galaxy with distinct chemistry from TriAnd. 

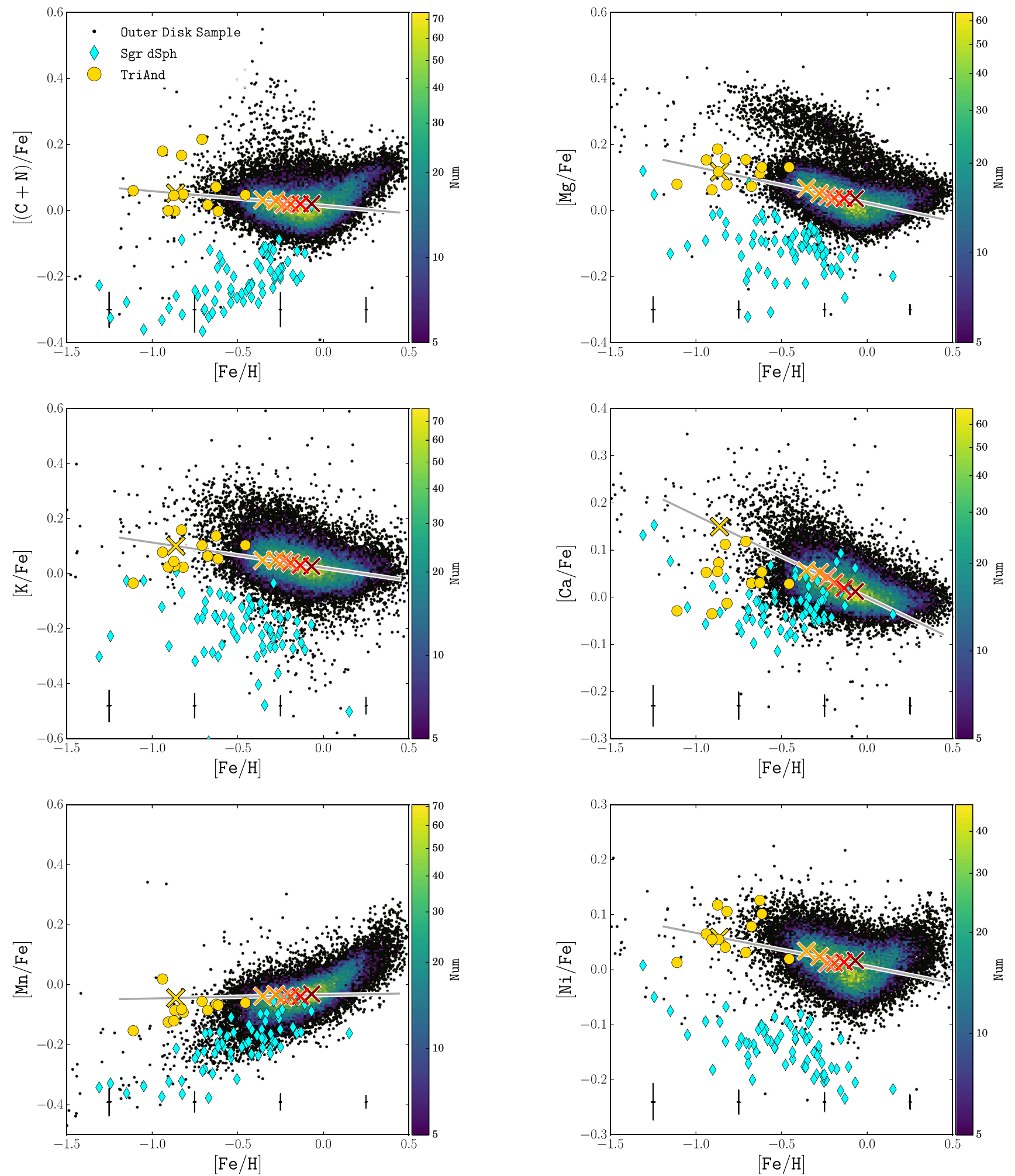

Figure 4. $[\mathrm{X} / \mathrm{Fe}]$ (for $(\mathrm{C}+\mathrm{N}), \mathrm{Mg}, \mathrm{K}, \mathrm{Ca}, \mathrm{Mn}$, and $\mathrm{Ni}$ ) vs. $[\mathrm{Fe} / \mathrm{H}]$ for the outer disk, TriAnd, and Sgr dSph samples (with colors and symbols as in Figure 2). The medians of the outer disk sample in $1 \mathrm{kpc}$-wide Galactocentric annuli are plotted as crosses that are colored according to Galactocentric radius, as in Figure 3. Linear parametric fits to $[\mathrm{X} / \mathrm{Fe}]-[\mathrm{Fe} / \mathrm{H}]$ medians from $R_{\mathrm{GC}}$ of $9 \mathrm{kpc}$ to $15 \mathrm{kpc}$ as a function of $R_{\mathrm{GC}}$ are shown (gray lines) and extrapolated out to $30 \mathrm{kpc}$, with $R_{\mathrm{GC}}=24 \mathrm{kpc}$ (the median Galactocentric radius of our TriAnd sample) marked as a gold cross. Typical uncertainties are shown as in Figure 3.

What about other potentially major accretion sources? Studies of metal-poor stars have uncovered two chemically distinct populations in the MW (e.g., Nissen \& Schuster 2010; Hawkins et al. 2015; Hayes et al. 2018). These two populations are (using the definitions from Fernández-Alvar et al. 2018;
Hayes et al. 2018) a "low-magnesium" halo population, thought to be accreted satellite galaxy debris, and a "highmagnesium" population, which continues the chemical trends of the thick disk and has the chemistry expected of the classical halo. The $[\mathrm{X} / \mathrm{Fe}]$ ratios of TriAnd stars in $(\mathrm{C}+\mathrm{N}), \mathrm{K}, \mathrm{Mn}$, and 
$\mathrm{Ni}$ are $0.2-0.4$ dex higher than the population of MW field stars thought to be accreted halo stars, and are also differentiated from the more classical halo population (and thick disk), which have high $\alpha$-element abundances.

The results here are in agreement with those of Bergemann et al. (2018), who argue for an association of TriAnd with the MW disk based on $\mathrm{O}, \mathrm{Na}, \mathrm{Mg}$, Ti, $\mathrm{Ba}$, and $\mathrm{Eu}$ abundances of eight TriAnd stars ${ }^{24}$ compared to MW, Fornax dSph, and Sgr dSph star samples. However, our conclusions are at odds with those reached by Chou et al. (2011), who proposed that TriAnd is more likely to be debris from a disrupted dwarf galaxy.

Chou et al. (2011) found a mean [Ti/Fe] ratio in their TriAnd sample of six stars (none of which overlap the Bergemann et al. sample or ours) about 0.2 dex lower than in Sgr dSph stars, and significantly lower than for their sample of MW stars from the solar neighborhood. These findings led to the original conclusion that TriAnd enriched slower than either population, consistent with expectations for a slowly enriching dwarf galaxy. However, this conclusion was largely drawn because half (three) of the stars in the Chou et al. TriAnd sample had $[\mathrm{Ti} / \mathrm{Fe}] \sim 0.5$ dex lower than their MW comparison sample despite (a) the remainder of their sample having [Ti/Fe] ratios consistent with the MW disk sample, and (b) Chou et al. finding their TriAnd sample to have $s$-process abundances in $\mathrm{La}$ and Y consistent with their MW disk trend. Unfortunately, Ti abundances cannot be reliably measured by ASPCAP currently (Hawkins et al. 2016; Souto et al. 2016), and we cannot study TriAnd Ti abundances here.

Given the disk-like $\alpha$-element abundances found for TriAnd stars by Bergemann et al. (2018, including disk-like Ti abundances), and those found for our TriAnd sample here, it seems that the lower $[\mathrm{Ti} / \mathrm{Fe}]$ ratios found by Chou et al. (2011) may not be representative of the $\alpha$-element abundances of TriAnd as a whole. Instead, the apparently considerably lower $[\mathrm{Ti} / \mathrm{Fe}]$ in three Chou et al. (2011) stars relative to the disk may be due to a variety of causes, including random measurement errors, systematic offsets between their TriAnd [Ti/Fe] and their adopted disk chemistries from the literature, or smallnumber statistics drawing from a TriAnd population with a potentially large intrinsic scatter in $\mathrm{Ti}$ abundances. Reconsidering that the Y, La, and half of the Ti abundances in the Chou et al. sample are consistent with MW disk abundance patterns, their results could be reinterpreted as supporting a disk origin.

In summary, we find that TriAnd is chemically distinct from the Sgr dSph, having $[\mathrm{X} / \mathrm{Fe}] \sim 0.1-0.4$ dex higher in $(\mathrm{C}+\mathrm{N})$, $\mathrm{Mg}, \mathrm{K}, \mathrm{Ca}, \mathrm{Mn}$, and $\mathrm{Ni}$, and is also distinct from the LMC in its $\mathrm{Ni}$ abundances, having $[\mathrm{Ni} / \mathrm{Fe}] \sim 0.3$ dex higher than the LMC stars observed by van der Swaelmen et al. (2013). On the other hand, while our TriAnd stars are typically more metal-poor $([\mathrm{Fe} / \mathrm{H}] \sim-0.8)$ than most outer disk stars sampled by APOGEE, TriAnd does appear to overlap in chemical space with the lowest metallicity stars $([\mathrm{Fe} / \mathrm{H}] \sim-0.7)$ known to lie in the outer regions of the disk. Moreover, linear extrapolation of each of the chemical gradients measured in the APOGEE outer disk sample to the Galactocentric radii of the TriAnd stars predicts abundances similar to those found in our TriAnd sample. These results support the proposition that TriAnd is associated with the outer disk of the MW and, if so, its large

\footnotetext{
${ }^{24}$ We have two stars in common with Bergemann et al. and the derived properties agree between the two studies within uncertainties and offsets of a typical size observed between APOGEE and optical studies (H. Jönnson et al. 2018 , in preparation)
}

distance from the midplane $(\sim 7 \mathrm{kpc})$ may be the result of a perturbation to the MW disk (as in Laporte et al. 2017).

If the TriAnd overdensity is indeed a feature of the MW disk, then that would imply that the disk extends to radii $\gtrsim 24 \mathrm{kpc}$ (i.e., the Galactocentric radius of our TriAnd sample), as suggested by Lopez-Corredoira et al. (2018). By inference, this greater MW disk would extend through the radii occupied by other Galactic anticenter overdensities (such as the Monoceros Ring) and lend greater weight to the notion that they, too, are parts of the MW disk.

The authors thank the referee for helpful comments. This research used TOPCAT (Taylor 2005). C.R.H. acknowledges the NSF Graduate Research Fellowship through grant DGE1315231. C.R.H. and S.R.M. acknowledge NSF grants AST1312863 and AST-1616636. Support for this work was provided by NASA through Hubble Fellowship grant \#51386.01 awarded to RLB by the Space Telescope Science Institute, which is operated by the Association of Universities for Research in Astronomy, Inc., for NASA, under contract NAS 5-26555 J.G.F.-T. is supported by FONDECYT No. 3180210. D.A.G.H. and O.Z. acknowledge support provided by the Spanish Ministry of Economy and Competitiveness (MINECO) under grant AYA-2017-88254-P. T.C.B. acknowledges partial support from grant PHY 14-30152 (Physics Frontier Center/JINA-CEE), awarded by the U.S. National Science Foundation.

Funding for the Sloan Digital Sky Survey IV has been provided by the Alfred P. Sloan Foundation, the U.S. Department of Energy Office of Science, and the Participating Institutions. SDSS-IV acknowledges support and resources from the Center for High-Performance Computing at the University of Utah. The SDSS web site is http://www. sdss.org.

SDSS-IV is managed by the Astrophysical Research Consortium for the Participating Institutions of the SDSS Collaboration including the Brazilian Participation Group, the Carnegie Institution for Science, Carnegie Mellon University, the Chilean Participation Group, the French Participation Group, Harvard-Smithsonian Center for Astrophysics, Instituto de Astrofísica de Canarias, The Johns Hopkins University, Kavli Institute for the Physics and Mathematics of the Universe (IPMU)/University of Tokyo, Lawrence Berkeley National Laboratory, Leibniz Institut für Astrophysik Potsdam (AIP), Max-Planck-Institut für Astronomie (MPIA Heidelberg), Max-Planck-Institut für Astrophysik (MPA Garching), MaxPlanck-Institut für Extraterrestrische Physik (MPE), National Astronomical Observatories of China, New Mexico State University, New York University, University of Notre Dame, Observatário Nacional/MCTI, The Ohio State University, Pennsylvania State University, Shanghai Astronomical Observatory, United Kingdom Participation Group, Universidad Nacional Autónoma de México, University of Arizona, University of Colorado Boulder, University of Oxford, University of Portsmouth, University of Utah, University of Virginia, University of Washington, University of Wisconsin, Vanderbilt University, and Yale University.

\section{ORCID iDs}

Christian R. Hayes (iD https://orcid.org/0000-0003-2969-2445 Steven R. Majewski (i) https://orcid.org/0000-0003-

2025-3147 
Rachael L. Beaton (ib https://orcid.org/0000-0002-1691-8217 Adrian M. Price-Whelan (i) https://orcid.org/0000-00030872-7098

Timothy C. Beers (ib https://orcid.org/0000-0003-4573-6233 Ricardo Carrera (i) https://orcid.org/0000-0001-6143-8151

Peter M. Frinchaboy (iD https://orcid.org/0000-0002-0740-8346

David L. Nidever (i) https://orcid.org/0000-0002-1793-3689

Alexandre Roman-Lopes (iD https://orcid.org/0000-00021379-4204

\section{References}

Abolfathi, B., Aguado, D. S., Aguilar, G., et al. 2018, ApJS, 235, 42 Bergemann, M., Sesar, B., Cohen, J. G., et al. 2018, Natur, 555, 334 Blanton, M. R., Bershady, M. A., Abolfathi, B., et al. 2017, AJ, 154, 28 Chou, M.-Y., Majewski, S. R., Cunha, K., et al. 2011, ApJL, 731, L30 Deason, A. J., Belokurov, V., Hamren, K. M., et al. 2014, MNRAS, 444, 3975 Fernández-Alvar, E., Carigi, L., Schuster, W. J., et al. 2018, ApJ, 852, 50 García Pérez, A. E., Allende Prieto, C., Holtzman, J. A., et al. 2016, AJ, 151,144

Gunn, J. E., Siegmund, W. A., Mannery, E. J., et al. 2006, AJ, 131, 2332 Hasselquist, S., Shetrone, M., Smith, V., et al. 2017, ApJ, 845, 162

Hawkins, K., Jofré, P., Masseron, T., \& Gilmore, G. 2015, MNRAS, 453, 758 Hawkins, K., Masseron, T., Jofré, P., et al. 2016, A\&A, 594, A43
Hayes, C. R., Majewski, S. R., Shetrone, M., et al. 2018, ApJ, 852, 49

Laporte, C. F. P., Johnston, K. V., Gómez, F. A., Garavito-Camargo, N., \& Besla, G. 2017, MNRAS, submitted (arXiv:1710.02538)

Li, T. S., Sheffield, A. A., Johnston, K. V., et al. 2017, ApJ, 844, 74

Lopez-Corredoira, M., Allende Prieto, C., Garzon, F., et al. 2018, A\&A, $612,8 \mathrm{~L}$

Majewski, S. R., Ostheimer, J. C., Rocha-Pinto, H. J., et al. 2004, ApJ, 615, 738

Majewski, S. R., Schiavon, R. P., Frinchaboy, P. M., et al. 2017, AJ, 154, 94 Martig, M., Fouesneau, M., Rix, H.-W., et al. 2016, MNRAS, 456, 3655

Martin, N. F., Ibata, R. A., \& Irwin, M. 2007, ApJL, 668, L123

Nidever, D. L., Holtzman, J. A., Allende Prieto, C., et al. 2015, AJ, 150, 173

Nissen, P. E., \& Schuster, W. J. 2010, A\&A, 511, L10

Price-Whelan, A. M., Johnston, K. V., Sheffield, A. A., Laporte, C. F. P., \& Sesar, B. 2015, MNRAS, 452, 676

Queiroz, A. B. A., Anders, F., Santiago, B. X., et al. 2018, MNRAS, 476, 2556

Rocha-Pinto, H. J., Majewski, S. R., Skrutskie, M. F., Crane, J. D., \& Patterson, R. J. 2004, ApJ, 615, 732

Sheffield, A. A., Johnston, K. V., Majewski, S. R., et al. 2014, ApJ, 793, 62

Souto, D., Cunha, K., Smith, V., et al. 2016, ApJ, 830, 35

Taylor, M. B. 2005, adass XIV, 347, 29

van der Swaelmen, M., Hill, V., Primas, F., \& Cole, A. A. 2013, A\&A, 560, A44

Xu, Y., Newberg, H. J., Carlin, J. L., et al. 2015, ApJ, 801, 105

Zasowski, G., Cohen, R. E., Chojnowski, S. D., et al. 2017, AJ, 154, 198

Zasowski, G., Johnson, J. A., Frinchaboy, P. M., et al. 2013, AJ, 146, 81 